\title{
Retraction Note to: Retinoic Acid Prevents Disruption of Blood-Spinal Cord Barrier by Inducing Autophagic Flux After Spinal Cord Injury
}

\author{
Yulong Zhou ${ }^{1,2} \cdot$ Binbin Zheng ${ }^{1,2} \cdot$ Libing Ye $^{2} \cdot$ Hongyu Zhang $^{2} \cdot$ Sipin Zhu ${ }^{1,2} \cdot$ Xiaomeng Zheng $^{2} \cdot$ Qinghai Xia $^{2}$. \\ $\mathrm{Zili} \mathrm{He}^{1,2} \cdot$ Qingqing Wang ${ }^{1,2} \cdot \operatorname{Jian} \mathrm{XiaO}^{2} \cdot$ Huazi Xu $^{1}$
}

Published online: 14 October 2020

(c) Springer Science+Business Media, LLC, part of Springer Nature 2020

\section{Retraction to: \\ Neurochemical Research (2016) 41: 813-825 https://doi.org/10.1007/s1106 4-020-03149-1}

The Editor-in-Chief has retracted this article [1] due to significant similarities with another paper by the first author which was under consideration at the same time and subsequently published [2]. In addition to some textual overlap in the Introduction, Results and Figure Legends, substantial similarities have been noted between the Fig. 1f of [1] and Fig. 1a of [2]. The level of replication between the two studies has led to overlap in the main findings. Therefore, this article is redundant.

Author Yulong Zhou does not agree to this retraction. Authors Jian Xiao and Huazi Xu have not responded to correspondence about this retraction. The journal has not been able to obtain email addresses for authors Binbin Zheng, Libing Ye, Hongyu Zhang, Sipin Zhu, Qinghai Xia, Xiaomeng Zheng, Zili He and Qingqing Wang.

The original article can be found online at https://doi.org/10.1007/ s11064-020-03149-1.

Jian Xiao

xfxj2000@126.com

$\triangle$ Huazi Xu

wzmcxu@163.com

1 Department of Orthopaedics, The Second Affiliated Hospital, Wenzhou Medical University, Wenzhou 325000, China

2 School of Pharmacy, Key Laboratory of Biotechnology and Pharmaceutical Engineering, Wenzhou Medical University, Wenzhou 325035, China

\section{References}

1. Zhou Y, Zheng B, Ye L et al (2016) Retinoic acid prevents disruption of blood-spinal cord barrier by inducing autophagic flux after spinal cord injury. Neurochem Res 41:813-825. https://doi. org/10.1007/s11064-015-1756-1

2. Zhou Y, Zhang H, Zheng B et al (2016) Retinoic acid inducedautophagic flux inhibits ER-stress dependent apoptosis and prevents disruption of blood-spinal cord barrier after spinal cord injury. Int J Biol Sci 12(1):87-99. https://doi.org/10.7150/ ijbs. 13229

Publisher's Note Springer Nature remains neutral with regard to jurisdictional claims in published maps and institutional affiliations. 